\title{
RADIALLY SYMMETRIC SOLUTIONS OF THE POISSON-BOLTZMANN EQUATION WITH A GIVEN ENERGY
}

Abstract. We consider the following problem:

$$
\Delta \Phi= \pm \frac{M}{\int_{\Omega} e^{-\Phi / \Theta}} e^{-\Phi / \Theta}, \quad E=M \Theta \mp \frac{1}{2} \int_{\Omega}|\nabla \Phi|^{2},\left.\quad \Phi\right|_{\partial \Omega}=0,
$$

where $\Phi: \Omega \subset \mathbb{R}^{n} \rightarrow \mathbb{R}$ is an unknown function, $\Theta$ is an unknown constant and $M, E$ are given parameters.

1. Introduction. We are interested in the motion of a system of Brownian particles confined to a thermally insulated container $\Omega \subset \mathbb{R}^{n}$ $(n=2,3)$. We assume that the particles move under the influence of mutual electric or gravitational interactions and are submitted to thermal diffusion (for physical background see [2], [8]).

Let $M, E$ denote the total charge (total mass) and energy of our system, respectively. It is known ([2]; [3], Ch. 10) that in the thermodynamical equilibrium the electric (gravitational) potential $\Phi$ of the system under consideration satisfies the Poisson-Boltzmann equation

$$
\Delta \Phi= \pm \frac{M}{\int_{\Omega} e^{-\Phi / \Theta}} e^{-\Phi / \Theta},
$$

where $\Theta$ is the temperature which can be evaluated from the equation of energy

$$
E=M \Theta \mp \frac{1}{2} \int_{\Omega}|\nabla \Phi|^{2}
$$

2000 Mathematics Subject Classification: 35J99, 35Q72, 82B05.

Key words and phrases: nonlinear elliptic problem, Poisson-Boltzmann equation. The preparation of this paper was supported by the KBN grant 2/P03A/011/19. 
The sign "-" ("+") in (1) corresponds to the Coulomb (gravitational) interactions. In (2) inversely, "-" corresponds to the gravitational interactions.

Using the notation $\varphi=\Phi / \Theta$ we transform (1)-(2) into

$$
\begin{aligned}
\Delta \varphi & = \pm \frac{M}{\theta} \frac{e^{-\varphi}}{\int_{\Omega} e^{-\varphi}}, \\
E & =M \theta \mp \frac{1}{2} \theta^{2} \int_{\Omega}|\nabla \varphi|^{2} .
\end{aligned}
$$

We suppose that at the boundary $\partial \Omega$ of $\Omega$, the potential $\varphi$ is constant. This assumption is physically reasonable in the case of Coulomb interactions (it means that the boundary is grounded) or for arbitrary interactions if the domain $\Omega$ is radially symmetric. The Poisson-Boltzmann equation is invariant with respect to the transformation $\varphi \mapsto \varphi+$ const, so we may put

$$
\left.\varphi\right|_{\partial \Omega}=0 \text {. }
$$

Multiplying (3) by $\varphi$ and integrating over $\Omega$ we get

$$
\|\nabla \varphi\|^{2}=\mp \frac{M}{\theta} \cdot \frac{1}{\int_{\Omega} e^{-\varphi}} \int_{\Omega} e^{-\varphi} \varphi .
$$

Hence the energy $E$ may be written in the form

$$
E=\frac{M \theta}{\int_{\Omega} e^{-\varphi}} \int_{\Omega} e^{-\varphi}\left(1+\frac{1}{2} \varphi\right) .
$$

Putting the temperature

$$
\theta=\frac{E}{M} \frac{\int_{\Omega} e^{-\varphi}}{\int_{\Omega} e^{-\varphi}\left(1+\frac{1}{2} \varphi\right)}
$$

into (3), we get a nonlocal elliptic equation for the potential $\varphi$,

$$
\Delta \varphi= \pm \frac{M^{2}}{E} \frac{\int_{\Omega} e^{-\varphi}\left(1+\frac{1}{2} \varphi\right)}{\left(\int_{\Omega} e^{-\varphi}\right)^{2}} e^{-\varphi} .
$$

For given $M$ and $E$ we are interested in the existence of solutions of (5), (7). We will call (5), (7) with the sign "-" the electric problem and with the sign "+" the gravitational problem. Note that in the case of electric interactions the energy is positive while for gravitational interactions it may also be negative. The equation (7) with boundary condition (5) is equivalent to our original problem (3)-(5) whenever $\Theta>0$. This is actually so in the case of electric interactions (since $E>0$ ) and for gravitational interactions in a two-dimensional ball. Note that we cannot expect this equivalence in higher dimensions for the gravitational case.

2. Two-dimensional radially symmetric case. We assume that $\Omega=\left\{x \in \mathbb{R}^{2}:|x| \leq 1\right\}$, the ball of radius one, and we look for radially 
symmetric solutions of $(5),(7)$. In this case our problem reads

$$
\begin{gathered}
\frac{1}{r}\left(r \varphi^{\prime}\right)^{\prime}(r)= \pm \frac{M^{2}}{E} \frac{\int_{0}^{1} s e^{-\varphi(s)}\left(1+\frac{1}{2} \varphi(s)\right) d s}{2 \pi\left(\int_{0}^{1} s e^{-\varphi(s)} d s\right)^{2}} e^{-\varphi(r)}, \\
\varphi^{\prime}(0)=0, \quad \varphi(1)=0 .
\end{gathered}
$$

Using the integrability of the Poisson-Boltzmann equation in the radially symmetric two-dimensional case we prove

TheOrem 1. The problem (8)-(9) has a unique solution.

P r o of. We will consider two cases.

(a) Electric problem (the sign "-" in (8)). The Poisson-Boltzmann equation

$$
\begin{gathered}
\frac{1}{r}\left(r \varphi^{\prime}\right)^{\prime}(r)=-C \frac{e^{-\varphi(r)}}{\int_{0}^{1} s e^{-\varphi(s)} d s}, \quad C>0, \\
\varphi^{\prime}(0)=0, \quad \varphi(1)=0,
\end{gathered}
$$

has the explicit solution $([1],[6])$

$$
\varphi_{C}(r)=2 \log \left(\frac{4+C-C r^{2}}{4}\right) .
$$

After simple calculations we obtain

$$
\begin{aligned}
& \left(\int_{0}^{1} s e^{-\varphi_{C}(s)} d s\right)^{-1}=\frac{4+C}{2} \\
& \int_{0}^{1} s e^{-\varphi_{C}(s)} \varphi_{C}(s) d s=\frac{4}{4+C}\left(1-\frac{4}{C} \log \left(1+\frac{C}{4}\right)\right) .
\end{aligned}
$$

Define

$$
V(C):=\frac{\int_{0}^{1} s e^{-\varphi_{C}(s)}\left(1+\frac{1}{2} \varphi_{C}(s)\right) d s}{2 \pi \int_{0}^{1} s e^{-\varphi_{C}(s)} d s} .
$$

Note that if $C$ satisfies $M^{2} / E=C / V(C)=: g(C)$ then $\varphi_{C}$ is a solution of (8)-(9).

Using (12), (13) we get

$$
\frac{2 \pi C}{2-\frac{4}{C} \log \left(1+\frac{C}{4}\right)}=g(C) .
$$

Obviously, $g(C)$ is a continuous, increasing function and $\lim _{C \rightarrow \infty} g(C)=\infty$, $\lim _{C \rightarrow 0^{+}} g(C)=0$. Hence for each $M \geq 0, E>0$ (as mentioned above, in the electric case $E>0)$ the equation $M^{2} / E=g(C)$ has a unique solution. 
(b) Gravitational problem (the sign "+" in (8)). It is easy to check that

$$
\varphi_{C}(r)=2 \log \left(\frac{4-C+C r^{2}}{4}\right), \quad C \in(0,4),
$$

is a unique solution of the problem $([1],[6])$

$$
\begin{gathered}
\frac{1}{r}\left(r \varphi^{\prime}\right)^{\prime}(r)=C \frac{e^{-\varphi(r)}}{\int_{0}^{1} s e^{-\varphi(s)} d s}, \\
\varphi^{\prime}(0)=0, \quad \varphi(1)=0 .
\end{gathered}
$$

After easy calculations we get

$$
\begin{aligned}
& \left(\int_{0}^{1} s e^{-\varphi_{C}(s)} d s\right)^{-1}=\frac{4-C}{2} \\
& \int_{0}^{1} s e^{-\varphi_{C}(s)} \varphi_{C}(s) d s=\frac{4}{4-C}\left(1+\frac{4}{C} \log \left(1-\frac{C}{4}\right)\right) .
\end{aligned}
$$

In this case the function $V$ defined above has the form

$$
V(C)=\frac{1}{\pi}\left(1+\frac{2}{C} \log \left(1-\frac{C}{4}\right)\right) .
$$

A function $\varphi_{C}$ is a solution of the gravitational problem (8)-(9) if and only if

$$
\frac{M^{2}}{E}=\frac{C}{V(C)}=\frac{2 \pi C}{2+\frac{4}{C} \log \left(1-\frac{C}{4}\right)}=: f(C) .
$$

It is easy to see that there exists $C_{1} \in(0,4)$ such that $f$ is increasing on $\left(0, C_{1}\right) \cup\left(C_{1}, 4\right)$ and $\lim _{C \rightarrow 0^{+}} f(C)=0, \lim _{C \rightarrow C_{1}^{-}} f(C)=\infty, \lim _{C \rightarrow C_{1}^{+}} f(C)$ $=-\infty$ and $\lim _{C \rightarrow 4^{-}} f(C)=0$. The graph of $f$ is shown in Figure 1 .

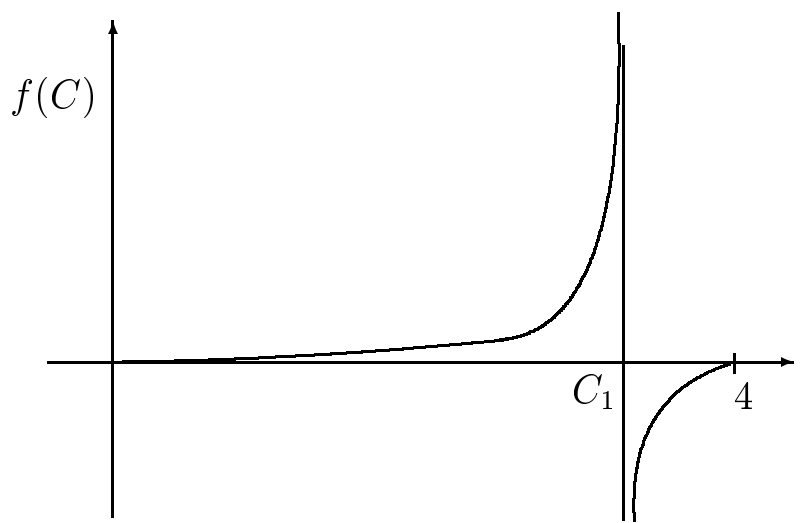

Fig. 1. The graph of the function $f(C)$ (eq. (16)) for $C \in(0,4)$ 
From Fig. 1 we see that the equation $M^{2} / E=f(C)$ has a unique solution for all $M>0, E \neq 0$.

REMARK. Similarly we may prove the existence of solutions of (8) in a ball of radius $R$. The auxiliary functions have the form

$$
\varphi_{C, R}(r)=2 \log \left(\frac{(4+C) R^{2}-C r^{2}}{4 R^{2}}\right), \quad C>0,
$$

in the case of electric interactions and

$$
\varphi_{C, R}(r)=2 \log \left(\frac{(4-C) R^{2}+C r^{2}}{4 R^{2}}\right), \quad C \in(0,4),
$$

in the gravitational case.

3. Radially symmetric problem (5), (7) in $\mathbb{R}^{n}$. We will consider the radially symmetric electric problem in a ball of radius one in $\mathbb{R}^{n}$.

Our problem reads

$$
\begin{gathered}
\frac{1}{r^{n-1}}\left(r^{n-1} \varphi^{\prime}\right)^{\prime}(r)=-\frac{M^{2}}{E} \frac{\int_{0}^{1} s^{n-1} e^{-\varphi(s)}\left(1+\frac{1}{2} \varphi(s)\right) d s}{\sigma_{n}\left(\int_{0}^{1} s^{n-1} e^{-\varphi(s)} d s\right)^{2}} e^{-\varphi(r)}, \\
\varphi^{\prime}(0)=0, \quad \varphi(1)=0
\end{gathered}
$$

where $\sigma_{n}$ is the area of the unit sphere in $\mathbb{R}^{n}$.

In the previous section we used the explicit form of solutions of the Poisson-Boltzmann equation in the two-dimensional case. Such formulas are not known in higher dimensions. To prove the existence for (17)-(18) we use the technique of sub- and supersolutions ([7]).

We start our considerations with the general electric problem (5), (7).

Let $\varphi_{C}$ be the unique solution ([4]) of the elliptic problem

$$
\begin{gathered}
\Delta \varphi_{C}=-C e^{-\varphi_{C}}, \quad C>0, \\
\left.\varphi_{C}\right|_{\partial \Omega}=0 .
\end{gathered}
$$

To prove the existence of solutions of (5), (7) we show that there exists a constant $C$ such that

$$
C=\frac{M^{2}}{E} \frac{\int_{\Omega} e^{-\varphi_{C}}\left(1+\frac{1}{2} \varphi_{C}\right)}{\left(\int_{\Omega} e^{-\varphi_{C}}\right)^{2}} .
$$

Integrating (19) over $\Omega$ we get

$$
\int_{\partial \Omega} \frac{\partial \varphi_{C}}{\partial \nu}=-C \int_{\Omega} e^{-\varphi_{C}}
$$

Using (22) we may write (21) in the form

$$
\frac{M^{2}}{E}=\frac{2\left(\int_{\partial \Omega} \partial \varphi_{C} / \partial \nu\right)^{2}}{C \int_{\Omega} \varphi_{C} e^{-\varphi_{C}}-2 \int_{\partial \Omega} \partial \varphi_{C} / \partial \nu}=: F(C) .
$$


Hence the problem of the existence of solutions of (5), (7) for $M, E>0$ is equivalent to showing that $F((0, \infty))=(0, \infty)$. We are able to prove it knowing the particular form of sub- and supersolutions of (19)-(20).

Lemma 1. For $\alpha<1 / 2$ and sufficiently large $C>0$ the function $\underline{\varphi_{C}}(r)=$ $2 \log \left(1+C^{\alpha}\left(1-r^{2}\right) /(2 n)\right)$ is a subsolution of $(19)-(20)$.

Pro of. After simple calculations we have

$$
\begin{aligned}
\Delta \underline{\varphi_{C}}= & -2 C^{\alpha} \frac{1+C^{\alpha}\left(1-r^{2}\right) /(2 n)}{\left(1+C^{\alpha}\left(1-r^{2}\right) /(2 n)\right)^{2}} \\
& -2 \frac{C^{2 \alpha}}{n^{2}} \frac{r^{2}}{\left(1+C^{\alpha}\left(1-r^{2}\right) /(2 n)\right)^{2}} .
\end{aligned}
$$

We have to show that $-\Delta \underline{\varphi_{C}} \leq C e^{-\underline{\varphi_{C}}}$. This is equivalent to the inequality

$$
2 C^{\alpha}\left(1+C^{\alpha} \frac{1-r^{2}}{2 n}\right)+2 \frac{C^{2 \alpha}}{n^{2}} r^{2} \leq C,
$$

which is obviously satisfied for $\alpha<1 / 2$ and sufficiently large $C$.

In a similar way we prove

Lemma 2. The function

$$
\overline{\varphi_{C}}=2 \log \left(1+C\left(1-r^{2}\right) /(2 n)\right)
$$

is a supersolution of (19)-(20) for all $C>0$.

Proof. We have

$$
\begin{aligned}
-\Delta \overline{\varphi_{C}}= & 2 C \frac{1+C\left(1-r^{2}\right) /(2 n)}{\left(1+C\left(1-r^{2}\right) /(2 n)\right)^{2}} \\
& +2 \frac{C^{2}}{n^{2}} \frac{r^{2}}{\left(1+C\left(1-r^{2}\right) /(2 n)\right)^{2}} .
\end{aligned}
$$

Putting (26) and $\overline{\varphi_{C}}=2 \log \left(1+C\left(1-r^{2}\right) /(2 n)\right)$ into the desired inequality $-\Delta \overline{\varphi_{C}} \geq C e^{-\overline{\varphi_{C}}}$ we get

$$
2+C \frac{1-r^{2}}{n}+2 \frac{C}{n^{2}} r^{2} \geq 1,
$$

which implies that $\overline{\varphi_{C}}$ is a supersolution of (19)-(20) for all $C>0$.

From Lemmas 1 and 2 we have

$$
\underline{\varphi_{C}}(r) \leq \varphi_{C}(r) \leq \overline{\varphi_{C}}(r) \text {. }
$$

Hence

$$
\left|\underline{\varphi_{C}^{\prime}}(1)\right|=2 \frac{C^{\alpha}}{n} \leq\left|\varphi^{\prime}(1)\right| \leq 2 \frac{C}{n}=\left|\overline{\varphi_{C}^{\prime}}(1)\right| .
$$


Thus

$$
F(C) \leq \int_{\partial \Omega}\left|\frac{\partial \varphi_{C}}{\partial \nu}\right| \leq \frac{2 C}{n},
$$

which implies that $\lim _{C \rightarrow 0^{+}} F(C)=0$.

Using (27) we may estimate $F(C)$ from below

$$
\begin{aligned}
& F(C)=\frac{\int_{\partial \Omega}\left|\partial_{\varphi} / \partial \nu\right|}{1+\frac{1}{2} C \frac{\operatorname{T} \Omega}{\partial \Omega} \varphi_{C} e^{-\varphi_{C}}} \frac{\partial \varphi_{C} / \partial \nu \mid}{\partial C^{\alpha} / n} \\
& \geq \frac{2 C^{\alpha} / n}{1+C^{1-\alpha}(n / 4) \int_{\Omega} e^{-\varphi_{C}} \varphi_{C}} \\
& =\frac{2}{n\left(C^{-\alpha}+(n / 4) C^{1-2 \alpha} \int_{\Omega} e^{-\varphi_{C}} \varphi_{C}\right)} \text {. }
\end{aligned}
$$

Asymptotically as $C \rightarrow \infty$,

$$
\int_{\Omega} e^{-\varphi_{C}} \varphi_{C}=\int_{0}^{1} 2 \log \left(1+C \frac{1-r^{2}}{2 n}\right) \frac{1}{\left(1+C^{\alpha}\left(1-r^{2}\right) /(2 n)\right)^{2}} d r \approx C^{\gamma}
$$

for some $\gamma<0$. Therefore $F(C) \rightarrow \infty$ as $C \rightarrow \infty$.

To show that $F((0, \infty))=(0, \infty)$ we prove

Lemma 3. The solution $\varphi_{C}$ of (19)-(20) depends continuously on $C$ in the supremum norm.

Proof. For $C>C_{0}$ the function $\bar{\varphi}=\left(C / C_{0}\right) \varphi_{C_{0}}$ is a supersolution of (19)-(20). In fact,

$$
-\Delta \frac{C}{C_{0}} \varphi_{C_{0}}=C e^{-\varphi_{C_{0}}} \geq C e^{-\left(C / C_{0}\right) \varphi_{C_{0}}} .
$$

Similarly, the function $\underline{\varphi}=\left(C / C_{0}\right) \varphi_{C_{0}}$ for $C<C_{0}$ is a subsolution of (19)-(20).

Moreover, for $C>C_{0}$ the function $\varphi_{C_{0}}$ is a subsolution of (19)-(20) and for $C<C_{0}$ the function $\varphi_{C_{0}}$ is a supersolution of (19)-(20).

Hence for $C_{1}<C_{0}<C_{2}$ we have

$$
\frac{C_{1}}{C_{0}} \varphi_{C_{0}} \leq \varphi_{C_{1}} \leq \varphi_{C_{0}} \leq \varphi_{C_{2}} \leq \frac{C_{2}}{C_{0}} \varphi_{C_{0}},
$$

which implies continuous dependence $\varphi_{C}$ on the parameter $C$.

We have shown

TheOREM 2. The problem (17)-(18) has a solution for all $M, E>0$.

Using the Schauder fixed point theorem and the estimates for the Green function and its derivatives we can prove 
Theorem 3. If $\Omega$ is a bounded domain in $\mathbb{R}^{n}(n \geq 3)$ with smooth boundary then the problem (5), (7) has a solution for sufficiently small values of $M^{2} /|E|$.

Pr o of. We define the operator $A: C^{0}(\bar{\Omega}) \rightarrow C^{0}(\bar{\Omega})$ by the formula

$$
A \varphi(x)= \pm \frac{M^{2}}{E} \frac{\int_{\Omega} e^{-\varphi}\left(1+\frac{1}{2} \varphi\right)}{\left(\int_{\Omega} e^{-\varphi}\right)^{2}} \int_{\Omega} G(x, y) e^{-\varphi(y)} d y,
$$

where $G(x, y)$ is the Green function for the Laplace operator. The estimates (cf. [5])

$$
\begin{aligned}
|G(x, y)| & \leq C(\Omega)|x-y|^{-(n-2)}, \\
\left|\nabla_{x} G(x, y)\right| & \leq C^{\prime}(\Omega)|x-y|^{-(n-1)}
\end{aligned}
$$

show that $A$ is a continuous and compact operator. To use the Schauder theorem we should show that $A$ transforms some ball $B_{R}(0) \subset C^{0}(\bar{\Omega})$ into itself.

In fact, for $\varphi \in B_{R}$ we have

$$
|A \varphi(x)| \leq \Gamma(\Omega) \frac{M^{2}}{|E|} e^{2 R}(1+R / 2),
$$

where $\Gamma(\Omega)=\sup _{x \in \Omega}\left|\int_{\Omega} G(x, y) d y\right|$. This implies that if

$$
\frac{M^{2}}{|E|} \leq \frac{1}{\Gamma(\Omega)} 2(3-2 \sqrt{2}) e^{-2(\sqrt{2}-1)}
$$

then $A \varphi \in B_{R}(0)$.

\section{References}

[1] C. Bandle, Isoperimetric Inequalities and Applications, Monographs Stud. Math. 7, Pitman, New York, 1980.

[2] P. Biler, A. Krzywicki and T. Nadzieja, Self-interaction of Brownian particles coupled with thermodynamic processes, Rep. Math. Phys. 42 (1998), 359-372.

[3] Ya. I. Frenkel', Statistical Physics, Izdat. Akad. Nauk SSSR, Moscow, 1948 (in Russian).

[4] D. Gilbarg and N. S. Trudinger, Elliptic Partial Differential Equations of Second Order, Springer, Grundlehren Math. Wiss. 224, Berlin, 2nd ed., 1983.

[5] M. Grüter and K.-O. Widman, The Green function for uniformly elliptic equations, Manuscripta Math. 37 (1982), 303-342.

[6] A. Krzywicki and T. Nadzieja, Some results concerning the Poisson-Boltzmann equation, Appl. Math. (Warsaw) 21 (1991), 365-272.

[7] C. V. Pao, Nonlinear Parabolic and Elliptic Equations, Plenum Press, New York, 1992. 
[8] R. F. Streater, A gas of Brownian particles in stochastic dynamics, J. Statist. Phys. 88 (1997), 447-469.

Tadeusz Nadzieja

Institute of Mathematics

Technical University of Zielona Góra

Podgórna 50

65-246 Zielona Góra, Poland

E-mail: T.Nadzieja@im.pz.zgora.pl
Andrzej Raczyński Mathematical Institute

University of Wrocław

Pl. Grunwaldzki 2/4

50-384 Wrocław, Poland

E-mail: aracz@math.uni.wroc.pl

Received on 3.2.2000;

revised version on 14.4 .2000

Added in proof (November 2000). Recently new results about the problem (3)-(5) were obtained. In the paper by P. Biler, J. Dolbeault, M. Esteban and G. Karch, Stationary solutions, intermediate asymptotics and large time behaviour of type II Streater's models, Adv. Differential Equations 6 (2001), 461-480, the authors proved the existence of solutions for electric interactions in an arbitrary domain. In the paper by P. Biler, J. Dolbeault, M. Esteban, P. A. Markovich and P. Nadzieja, Steady states for Streater's energy-transport models of self-gravitating particles (preprint), some partial results were obtained in the case of gravitational interactions. 